\title{
Lth Band Filter Design and Performance Analysis for WIMAX Application
}

\author{
Ayushi Gagneja \\ M.E Student \\ Department of Electronics \&Communication, \\ National Institute of Technical Teachers Training \& \\ Research, Chandigarh, India -160019
}

\author{
Rajesh Mehra, PhD \\ Assistant Professor \\ Department of Electronics \&Communication, \\ National Institute of Technical Teachers Training \& \\ Research, Chandigarh, India -160019
}

\begin{abstract}
Nyquist Filters which are generally used for equalization of video signals, are an essential part of communication systems. In this paper two Nyquist filters have been compared and analyzed having different L-bands. Its implementation can be easily found in the developing communication systems, but here results are claimed for WIMAX applications. The proposed design and analysis have been developed with the help of MATLAB with same order, sampling frequency, transition width and roll off factor as per the WIMAX specifications. The filters are then decimated by a decimation factor of 4 . The cost implementation of both has been taken into consideration and a result is drawn which concludes that Nyquist filter two bands is much more cost effective as compared to the Nyquist filter with three bands.
\end{abstract}

\section{General Terms}

Performance Analysis of Lth band Filter for WIMAX

\section{Keywords}

DDC, DUC, Digital Signal Processing, FIR filter, MATLAB, Signal Sampling, WIMAX

\section{INTRODUCTION}

The communication systems have seen immense progress in recent times. Its vast evolution has allowed new functionalities and higher performance of devices. In today's world, Digital signal processing which uses digital representation of signals for transmission, storage and reception have to face various challenges like how to lower the circuit complexity as well as reducing the cost of products. Now-a-days all the communication devices including cell phones, video phones, satellite phones, portable computers etc. require DSP algorithms to overcome the earlier difficulties [1]. Digital conversion is a fundamental part of many digital radio systems. These systems though being a fast medium, still undergo many losses, hence electronic devices called digital filters have technologically advanced as a resilient option for eliminating noise, shaping spectrum, and reducing inter-symbol interference in communication architectures. To obtain high performance levels, which were these digital filters come with precise reproducibility, thus becoming popular among engineers. Multi rate systems are the main building blocks of any communication system. Their main function is alteration of rate of the discrete-time signals, by the process of addition or deletion of a portion of the signal samples. These filters are proposed to be enormously powerful structures which concurrently perform digital filtering, spectral translation, interpolation, and decimation in both non-recursive and recursive structures [2]-[5]. Multirate systems also play a major role in communication systems. Multirate filters offer relatively lower sampling rates thereby reducing the order of system along with minimizing the hardware requirements, hence reducing the complexity of the product.

In this paper, work has been done using a Nyquist filter which is the electronic filter used in TV receivers to equalize the video characteristics. It's basic function being equalizing the low and high frequency components of the VF (video signal) signal. It is used before demodulation and acts as a low-pass filter with $6 \mathrm{~dB}$ suppression at the intermediate frequency (IF) carrier. Thus suppressing the levels of double sideband portion of the VF signal and reconstructing the original band characteristic at the output of the demodulator [6]. These play an essential role in the generation of $\mathrm{n}$ band limited pulse for minimum inter-symbol interference in wired as well as wireless communication systems. It has found its applications mainly as the pulse shaping filter. Also low pass Nyquist filter can be used as the raised cosine Nyquist fir filter. The L-bands are varied for the study i.e. the values are kept as 2 and 3 and hence the performance is analyzed for both in case of WIMAX digital down convertor.

\section{WIMAX}

IEEE802.16 popularly known as WIMAX (worldwide interoperability for microwave access, is a family of wireless communications standards initially designed to provide enhanced data rates i.e. 30-40 Megabit-per-second. The worldwide interoperability for microwave access standard is an emerging technology with significant potential that is poised to revolutionize the broadband wireless internet access market. It states air limitations of fixed broadband wireless access (BWA) systems supporting multimedia services. WIMAX can provide Internet access across regions of large boundaries [6]-[7].

The WIMAX standard requires a sharp transition band between $4.75 \mathrm{MHz}$ and $5.45 \mathrm{MHz}$. The stop band attenuation for practical implementations is usually required to in the order of $90 \mathrm{~dB}$, for successful operation in interference prone situations [8]. The evolution of internet lately has led to the increase in demand of high speed internet access. Broadband wireless access (BWA) has gained a lot of fame as an alternative wireless technology to DSL lines and cable modems [3]. The configuration of DUC or DDC is subject to conversion ratio required for WIMAX systems the conversion ratio is typically 8 to 10 [9]. The low conversion factor DDC or DUC processes is characteristically constructed using FIR filters only and for greater conversion rates CIC filters are normally used. Designer of WIMAX system needs to meet numerous requirements like processing speed, flexibility and time to market and it is these inflexible requirements that ultimately drive the hardware platform [4]. 


\section{DIGITAL DOWNCONVERTOR}

Digital electronics plays a vital role in wireless communication due to extensive usage of digital representation of data for communication. In DSP, a digital down converter (DDC) converts a digitized real signal positioned at an intermediate frequency (IF) to a base band complex signal positioned at zero frequency. Sampling rate conversion causes aliasing and imaging because the process involved is resampling [10]. Also, it decimates to a lower sampling rate, allowing follow-on signal processing by lower speed processors given in Figure1. So to eliminate their effects, resampling requires filtering.

Fs

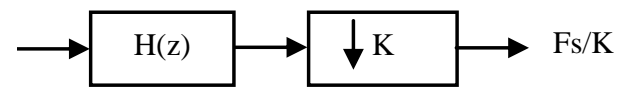

Figure1. Conceptual view of a decimation Filter

It receives an incoming digital IF signal and modifies the signal into a baseband complex signal, resulting in an in-phase signal and also a Quadrature signal as output. The design of the DDC can be implemented using FPGAs [11].The digital down converter (DDC) performs the reverse function compared to that of a DUC. It converts a signal from the IF band to the base band [8].

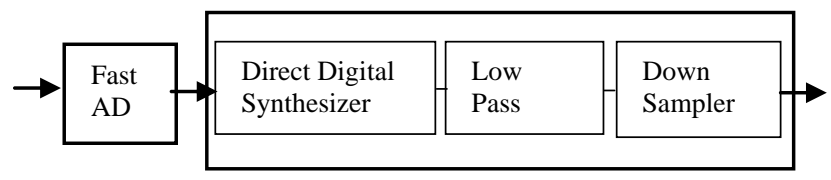

Figure 2: Block diagram of DDC

DDC consists of three sub-parts: a direct digital synthesizer (DDS), a low-pass filter (LPF), and a down sampler as presented in Figure2. Direct digital synthesis is a configuration by which a sinusoidal signal is formed by the use of digital numbers which control the input of a sinusoidal look-up table [12]. It produces a complex sinusoid at the intermediate frequency. Multiplication of the intermediate frequency and the input signal generates images positioned at the sum and difference of the frequencies. The low pass filters pass the difference i.e. baseband frequency while declining the sum frequency image, triggering in a complex baseband representation of the original signal. Hence the most common and preferred choice is a FIR filter for low amounts of decimation and the best architecture is considered to be the one which can be accommodated in hardware consuming less area and power as compared to various other designs available in the market [4].

The process of decimation is clearly illustrated in the Figure given below. In this example a sinusoidal wave in the original form as well as decimated form can be seen.
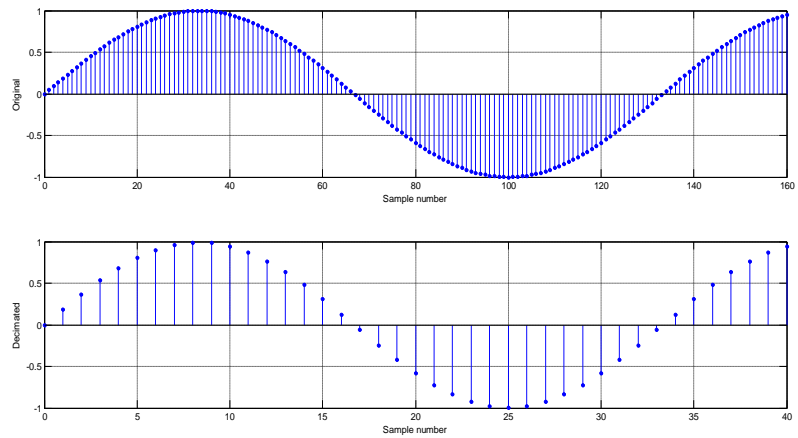

Figure 3: Example of Decimator

DDC performs the two essential functions. First one is frequency translation and other is channel filtering. Herein, a mixer and local oscillator execute the frequency translation. The local oscillator comprises of a digital phase accumulator that progresses each clock by an increment equivalent to the tuning frequency. A sine/cosine lookup table alters the phase angle of the accumulator to the digital voltage value of the sinusoid. The output sinusoid is relative to the phase increment or frequency setting. This block is a termed as The Numerically Controlled Oscillator (NCO). The mixer entails two digital multipliers that accept complex sine/cosine outputs from the local oscillator and digital samples of the receiver input signal produced by an $\mathrm{A} / \mathrm{D}$ converter. The filter is a complex low-pass digital filter with two parallel I and Q arms whose coefficients are programmed for a pass band equal to the channel bandwidth [11].

The DDC utilize the lower resource consumption, low power consumption, low circuit intricacies and high speed in wireless communication systems to ease the cost of main design. These find their major applications in the field of application specific integrated circuits or the so called field programmable gate arrays or commonly known as FPGAs. The main DDC manufacturers include giant companies' names including Xilinx, Altera, Texas Instruments and many more.

\section{DESIGN SIMULATIONS}

A Nyquist filter for L-bands is used for decimation as per the requirement for WIMAX applications. Practical digital communication systems use a roll off factor between 0.1 and 0.5 . In this paper the roll off factor is selected as 0.25 . The stop band attenuation is required in approximation of $90 \mathrm{~dB}$ while a sharp transition band is set between $4.75 \mathrm{MHz}$ and 5.45 MHz. For further analysis the order is fixed at 10 . Sampling frequency for WIMAX is known to be $91.392 \mathrm{MHz}$. Magnitude and Phases analysis have been given in Figure4 for the value of $\mathrm{L}=2$ and decimation factor of 4 . This filter can also be called Hand Band Low Pass Filter. As for the Impulse Response of the filter, it shows that an alternative zero occurs on both sides of the graph taking $\mathrm{Fc}=0 \mathrm{~Hz}$ as the center, which can be easily seen in Figure5. The step response of the filter shows variation from positive to negative and then again reach back to positive within the range of $0-40 \mathrm{~dB}$ as in the Figure6. 


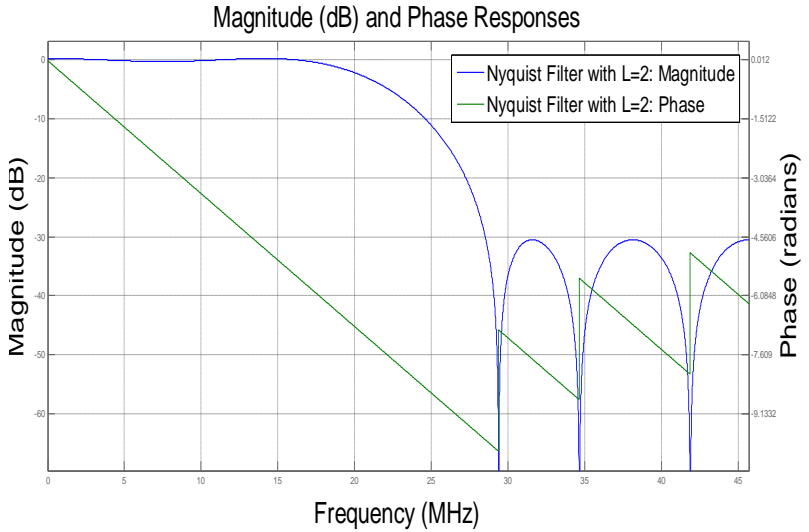

Figure 4: Magnitude and Phase Analysis of $1^{\text {st }}$ Filter

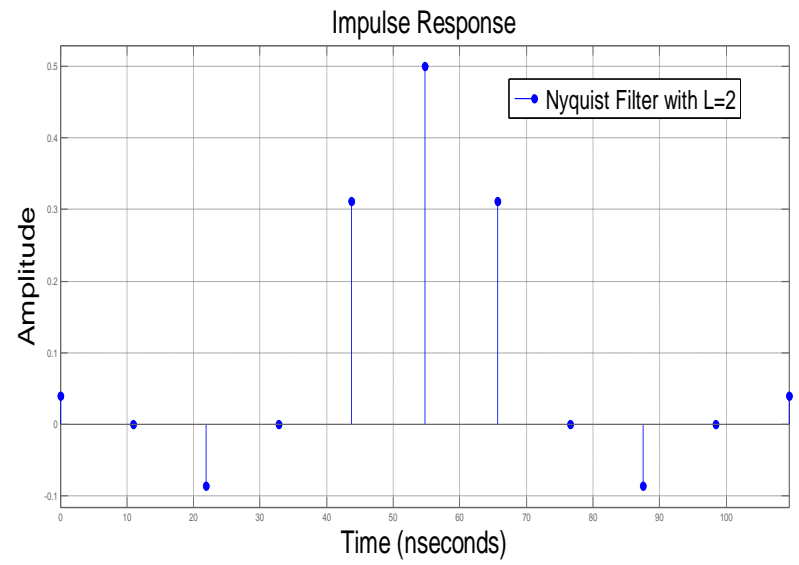

Figure 5: Impulse Response of Nyquist Filter with $L=2$

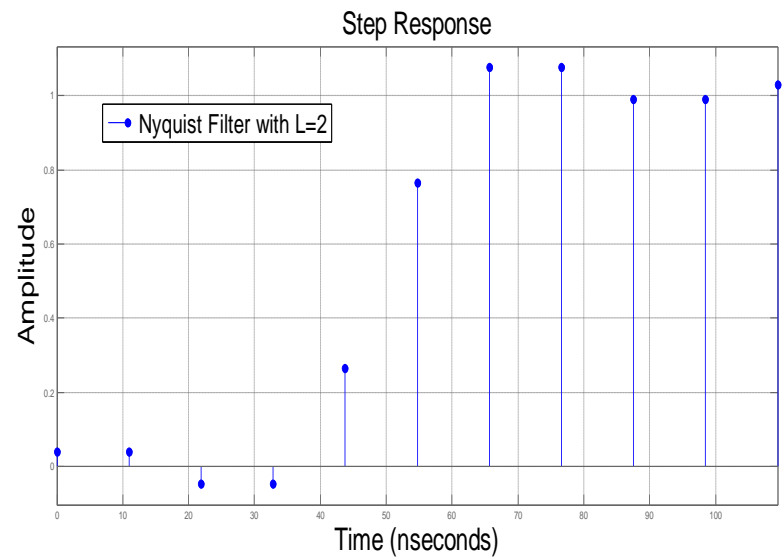

Figure 6: Step Response of Nyquist Filter with $L=2$

Another Nyquist filter for $\mathrm{L}=3$ is analyzed for WIMAX. It has the same sampling frequency of $91.392 \mathrm{MHZ}$, as the previous filter. Decimation factor and roll off factor also remains same, i.e. 4 and 0.25 respectively, while transition band is kept constant at $4.75 \mathrm{MHz}$.

Magnitude and Phases analysis have been given in Figure7 for the value of $\mathrm{L}=3$. The Impulse Response of the filter shows that a zero occurs after every second frequency component on both sides of the graph taking $\mathrm{Fc}=2.2 \mathrm{MHz}$ as the center, which can be easily seen in Figure8. As for the step response of the filter shows variation from negative to positive within the range of $20-40 \mathrm{~dB}$ as can be seen in the Figure9.

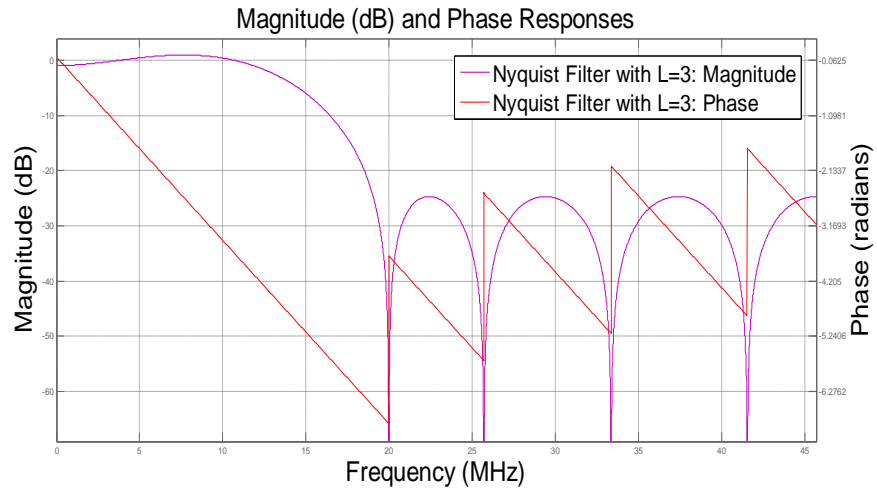

Figure 7: Magnitude and Phase Analysis of $2^{\text {nd }}$ Filter

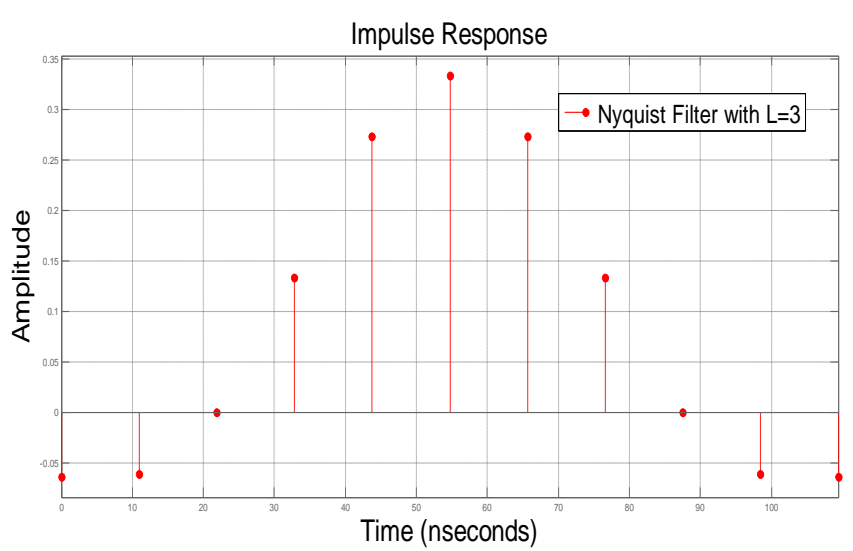

Figure 8: Impulse Response of Nyquist Filter with $L=3$

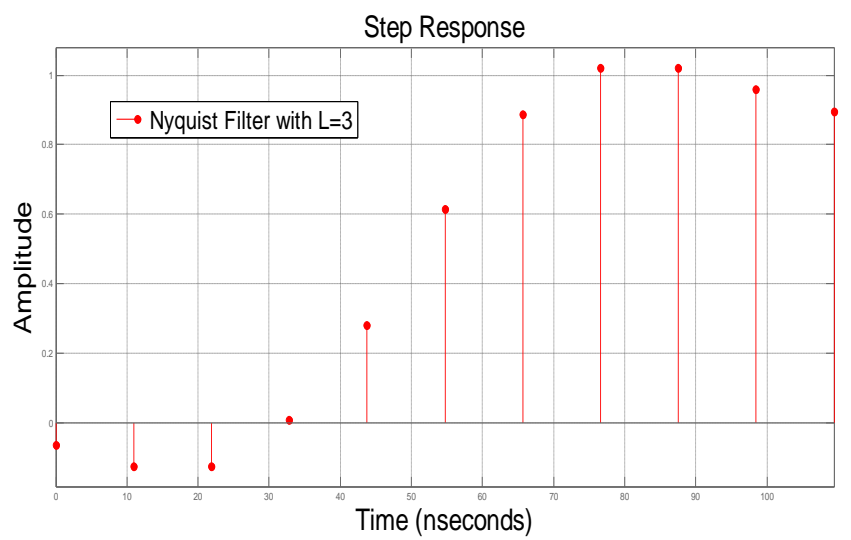

Figure 9: Step Response of Nyquist Filter with $L=3$

\section{PERFORMANCE ANALYSIS}

The Nyquist filters have been developed especially for WIMAX based applications. Digital down conversion is carried out in both the filters with same decimation factor of 4 and also same order of 10 . The sampling frequency, roll off factor as well as transition width is kept same for both the Nyquist filters so that the various performance parameters can be compared on an equivalent basis and hence comparison results can be obtained. In this paper magnitude response comparison has been made of the Nyquist filters with $\mathrm{L}=2$ and $\mathrm{L}=3$, with a decimation factor of 4 and order equal to 10 , in case of WIMAX applications, as shown in the Figure10.

As for the impulse response, both results have been brought up on a single plot for comparison. In the Figure11, blue plot represents Nyquist filter with $\mathrm{L}=2$ which has zero at every alternate frequency around $\mathrm{Fc}$ on both the sides, while the green plot which represents Nyquist filter with $\mathrm{L}=3$ has zero 
at every second frequency on both the sides around the same Fc.

Step response comparison has also been made in Figure12. In this the blue plot $(\mathrm{L}=2)$ has positive-negative-positive relation in $0-40 \mathrm{~dB}$ range, while in the same range, the green plot $(\mathrm{L}=3)$ travels from negative to positive.

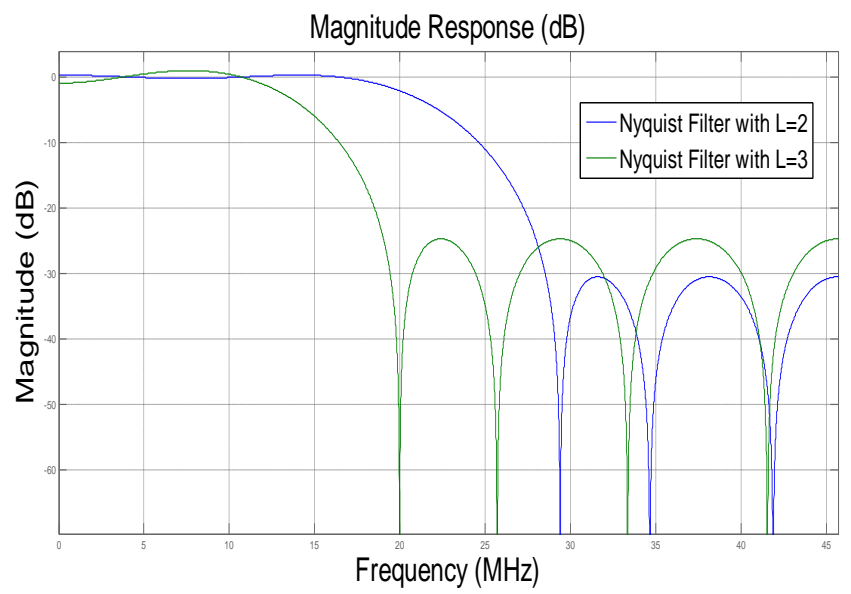

Figure 10: Magnitude Comparison of both filters

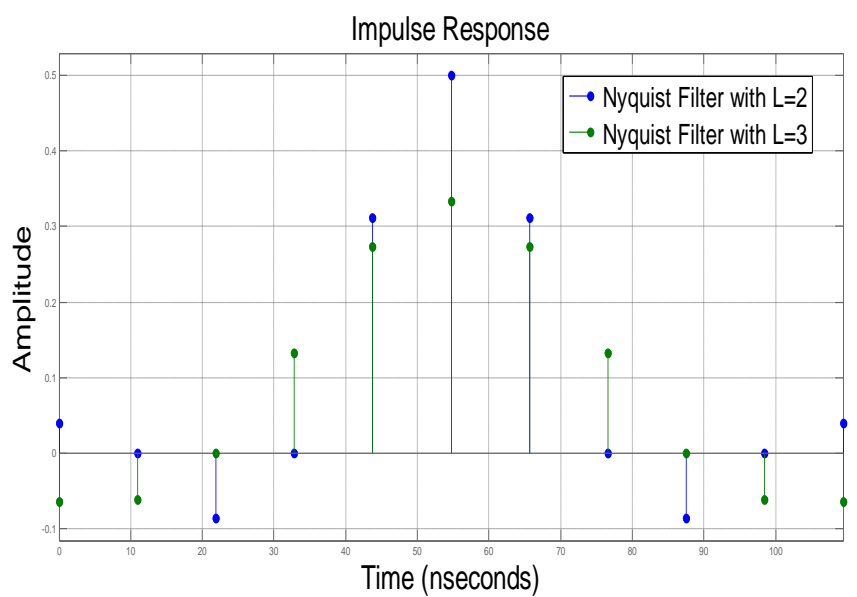

Figure 11: Impulse Response Comparison of both filters

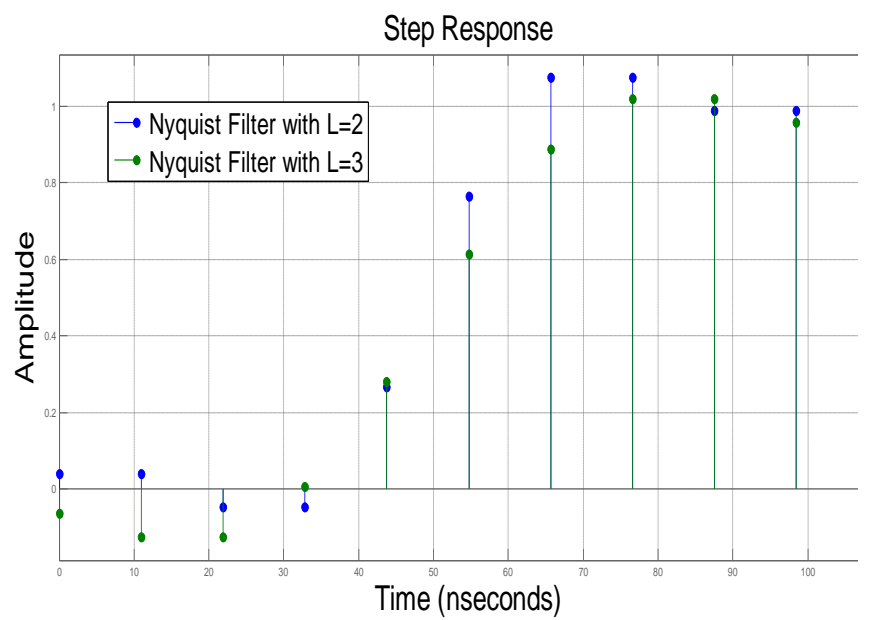

Figure 12: Step Response Comparison of both filters

The cost of both the filters along with resource consumption of both the Nyquist filters are compared and results have been drawn in Table1.
Table 1: Resource Comparison of both the Nyquist Filters

\begin{tabular}{|l|l|l|}
\hline $\begin{array}{l}\text { Implementation } \\
\text { Cost }\end{array}$ & $\begin{array}{l}\text { Nyquist Filter } \\
\text { with L=2 }\end{array}$ & $\begin{array}{l}\text { Nyquist Filter } \\
\text { with L=3 }\end{array}$ \\
\hline No. of Multipliers & 7 & 9 \\
\hline No. of Adders & 6 & 8 \\
\hline No. of States per & 1.75 & 8 \\
\hline $\begin{array}{l}\text { Multiplication } \\
\text { Input sample }\end{array}$ & 2.25 \\
\hline $\begin{array}{l}\text { Additions per Input } \\
\text { sample }\end{array}$ & 1.5 & 2 \\
\hline
\end{tabular}

Hence the requirement of multipliers and adders are less in case of Nyquist Filter with $L=2$, for same number of states 8 , decimation factor 4 and order 10.It is also observed that both the Nyquist filters same poly phase length and filter length for same decimation factor 4 and same order 10. Both the filters have linear phase and stability.

\section{CONCLUSION}

The proposed design is observed to perform better with the use of Nyquist Filter having L-bands value equal to 2 . MATLAB tool used for its implementation shows a significant improvement in terms of number of multipliers and adders required. Moreover the additions per sample and the multiplications per sample are far less than the other discussed filter. Hence it improves the speed and computational complexity of the system.

\section{ACKNOWLEDGMENTS}

The author would like to thank the Director and Head of Electronics and Communication Engineering Department, National Institute of Technical Teachers Training and Research, Chandigarh, India. This research paper wouldn't have been possible without their guidance and support, their inspirations, support and helpful suggestions were like a backbone in the completion of this research work.

\section{REFERENCES}

[1] Rajesh Mehra, "Reconfigurable Optimized WCDMA DDC for Software Defined Radios", Journal of Selected Areas in Telecommunications (JSAT), pp. 1-6, 2010.

[2] K.Shanthi, Dr.B.K.Madhavi, "Implementation of Optimized Cascaded Integrator Comb Filters for Digital Up, Down Conversions", International Journal of Science, Engineering and Technology Research (IJSETR), Volume 4, Issue 7, pp. 2558-2562, 2015.

[3] Sumeet Prashar, Rajesh Mehra, "Design and Analysis of Multirate Filter for WIMAX Application", International Journal of Engineering Research and Development, Volume 5, Issue 2, pp. 60-63, 2012.

[4] S.Meenakshi, Mrs.P.Uma, "Digital Up/Down Converter Design \& VLSI Implementation for Interpolators/Decimators", International Journal of Emerging Technology \& Research, Volume 1, Issue 4, pp.876-80, 2014. 
[5] Rajeev Ratan, Dr. Sanjay Sharma and Dr. Amit K. Kohli, "Effect of Compensation and Arbitrary Sampling in interpolators for Different Wireless Standards on FPGA Platform", Research Journal of Applied Sciences, Engineering and Technology, Volume 6, Issue 4, pp.609621, 2013.

[6] Charanjit Singh, Dr. Manjeet Singh Patterh and Dr. Sanjay Sharma, "Design of programmable digital down converter for WIMAX", Indian Journal of Science and Technology Volume 2, Issue 3, pp.20-21, 2009.

[7] "Digital Up/Down Converter (DDC/DUC) for WIMAX Systems" by Lattice Semiconductor, pp. 1-8, 2008.

[8] Mathworks, "Users Guide Filter Design Toolbox 4", 4.34.80, 2007
[9] "Accelerating DUC \& DDC System Designs for WIMAX" by Altera Corporation, Version 2.2, pp. 1-6, 2007.

[10] Rajesh Mehra, Lajwanti Singh, "FPGA based Speed Efficient Decimator using Distributed Arithmetic Algorithm", International Journal of Computer Applications, Volume 80, Issue 11, pp. 37-38, 2013

[11] Maruthi G.B, Saleem Malik, S.P Prashanth Kumar, Pratap M.S, "Implementation of High Performance DUC and DDC for Software Defined Radio Applications", International Journal of Computer Applications, Volume 110, Issue 6, pp.23-25, 2015.

[12] Rajesh Mehra, Bindiya Kamboj, "Efficient FPGA Implementation of Direct Digital Frequency Synthesizer for Software Radios", International Journal of Computer Applications, Volume 37, Issue 10, pp. 25-26, 2012. 